\title{
Pion Production in High-Energy Nucleus-Nucleus Collisions
}

\author{
J. W. Harris, G. Odyniec, H. G. Pugh, L. S. Schroeder, and M. L. Tincknell \\ Nuclear Science Division, Lawrence Berkeley Laboratory, University of California, Berkeley, California 94720 \\ W. Rauch and R. Stock \\ Fachbereich Physik, University of Frankfurt, Frankfurt, West Germany \\ R. Bock, R. Brockmann, A. Sandoval, and H. Ströbele \\ Gesellschaft für Schwerioneneforschung, Darmstadt, West Germany \\ R. E. Renfordt and D. Schall \\ University of Heidelberg, Heidelberg, West Germany \\ D. Bangert ${ }^{(a)}$ \\ University of Marburg, Marburg, West Germany \\ J. P. Sullivan and K. L. Wolf \\ Texas A\&M University, College Station, Texas 77843 \\ and \\ A. Dacal, C. Guerra, ${ }^{(b)}$ and M. E. Ortiz \\ Instituto de Fisica, Universidad Nacional Autónoma de México, Mexico City, 21 Distrito Federal, Mexico \\ (Received 18 August 1986)
}

\begin{abstract}
Negative-pion multiplicity $\left\langle n_{\boldsymbol{z}}\right\rangle$ was measured over the range of participant nucleon number $80 \leq A \leq 270$ for incident energies from 530 to $1350 \mathrm{MeV} /$ nucleon in the La+La system. The $\left\langle n_{\boldsymbol{x}}\right\rangle$ is proportional to $A$ and increases linearly with the c.m. energy. Thermal and potential energies, and temperatures of the maximum-density phase of the collision are extracted from the data. The results require a stiff nuclear-matter equation of state.
\end{abstract}

PACS numbers: $25.70 . \mathrm{Np}$

Determining the response of nuclear matter to extreme changes in temperature and density is important to the understanding of effective baryon-baryon interactions ${ }^{1}$ as well as supernova explosions ${ }^{2}$ and neutron-star formation $^{3}$ and structure. ${ }^{4}$ Recent studies of relativistic nucleus-nucleus collisions ${ }^{5-7}$ have shown that particle production, in the form of pions and kaons, provides information on the kinetic energy available in the highdensity stage of the reaction. The potential, and thus compressional, energy can then be determined by use of the total initial energy and energy conservation. Others $^{8,9}$ have studied the decompression stage of the reaction, finding that some of the compressional energy reappears in asymmetric flow of matter. Since initial efforts $^{5,6}$ to extract the compressional energy from pion multiplicities employed the relatively light $\mathrm{Ar}+\mathrm{KCl}$ system and led to a surprisingly stiff equation of state, it is important to extend this study to heavier nuclei to investigate the degree to which surface effects and other size phenomena may affect the conclusions.

Negative-pion production in collisions of ${ }^{139} \mathrm{La}+{ }^{139} \mathrm{La}$ was studied in the Bevalac Streamer Chamber at incident laboratory energies of $530,740,990,1200$, and $1350 \mathrm{MeV} /$ nucleon. Minimum-bias, semicentral, and central trigger configurations ${ }^{10}$ were employed at each incident energy. In a geometric model, these triggers correspond to impact parameters $b<b_{\max }, b<0.73 b_{\max }$, and $b<0.24 b_{\max }$, respectively. Extrapolation to zero impact parameter as done previously ${ }^{6}$ with the lighter $\mathrm{Ar}+\mathrm{KCl}$ system is difficult for the heavy system because of the presence of a larger number and variety of nuclear fragments. Only after implementation of a 384-element scintillator array, covering angles $\theta_{\mathrm{lab}}<18^{\circ}$, was this extrapolation possible. It provided position, charge, and time-of-flight information allowing extraction of the total spectator charge and, by subtraction, the number of participant protons in each event. The negative-pion multiplicities were determined by our scanning and measuring tracks on film. The participant and pion information were then correlated event by event.

Projectile spectators were identified in the array by pulse height, emission angle, and time of flight. The minimum-bias trigger, which selects mostly peripheral collisions dominated by spectator emission, was used at each incident energy to determine the spectator windows on these quantities: (a) The $d E / d x$ spectrum which yields projectile spectator charge was calibrated with fragmentation products from $\mathrm{Ne}$ and $\mathrm{La}$ beams. Unit 
charge identification was achieved for fragment charges $1 \leq Z \leq 10$. (b) The projectile-spectator angle window was defined to be the region centered about beamvelocity $Z=1,2$ fragments containing $90 \%$ of the charged particles in the minimum-bias data. The Fermi momentum of the projectile nucleons implied by this procedure was approximately $240 \mathrm{MeV} / c$. (c) The projectile-spectator time-of-flight window was defined to correspond to $90 \%$ of the time-of-flight spectrum of charged particles observed in the scintillators in the minimum-bias data.

Since there is no complete separation in phase space of participants and spectators at these incident energies, a Monte Carlo simulation was carried out to ascertain the efficiency of this procedure for the selection of spectators in the semicentral and central data. A cascade code ${ }^{11}$ was used to generate events corresponding to the three trigger modes. The participants and spectators, which are distinguished in this microscopic model, provided characteristic position and time-of-flight distributions at the downstream scintillator array after simulation of their trajectories through the streamer-chamber magnetic field. By this procedure, a final efficiency factor was determined and applied to the data. ${ }^{12}$ The projectile spectator charge was transformed to participant nucleon number by our assuming (a) the total (projectile+target) spectator charge $Q_{\text {spec }}$ to be twice the measured projectile spectator charge ( $A+A$ collisions), and (b) the total participant nucleon number to be $(A / Z)(2 Z$ $\left.-Q_{\text {spec }}\right)$, where $Z$ and $A$ are the nuclear charge and mass of La.

The streamer-chamber $\pi^{-}$data were corrected for losses in the beam entry pipe $\left(\theta_{\text {lab }}>130^{\circ}\right)$, identification losses along the magnetic field direction, losses due to

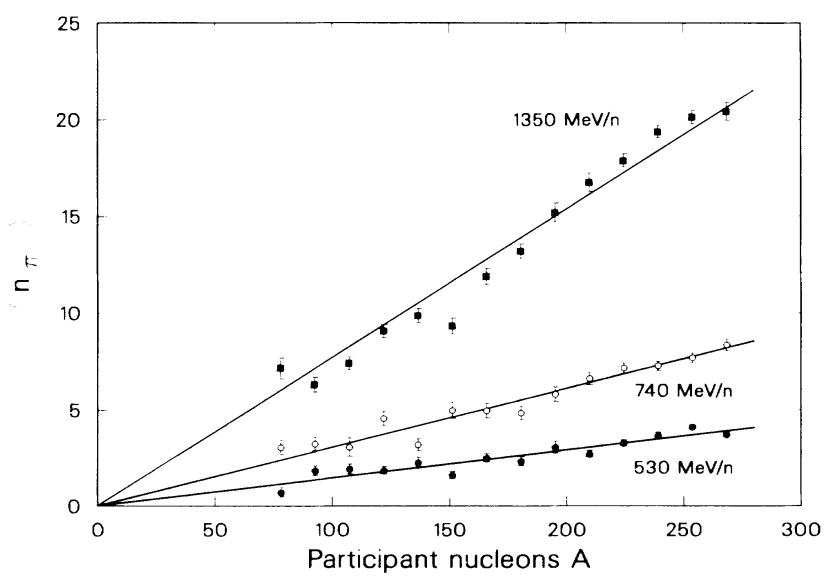

FIG. 1. The mean pion multiplicity as a function of the number of participant nucleons $A$ in the $\mathrm{La}+\mathrm{La}$ reaction at three incident laboratory energies. The lines are straight-line fits to the data points. stopping in the target $(p<50 \mathrm{MeV} / c)$, and for target conversion of gammas (from neutral-pion decay) into electron-position pairs where electrons are misidentified as negative pions. These corrections were $+(3.1$ to $4.5) \%$, $+(13$ to 15$) \%$, $+(1.4$ to 2.5$) \%$, and $-(5.8$ to $6.3) \%$, respectively, depending upon the incident energy.

The observed negative-pion multiplicity $\left\langle n_{\pi}\right\rangle$ is displayed in Fig. 1 as a function of participant nucleon number for the semicentral and central trigger modes at three different incident energies. It is proportional to the participant nucleon number $A$ at each incident energy and the slopes are the same as those observed ${ }^{6}$ for $\mathrm{Ar}+\mathrm{KCl}$ at the same incident energies.

The total pion multiplicity was derived from $\left\langle n_{\pi}\right\rangle=2.35\left\langle n_{\pi}\right\rangle$, where the factor ${ }^{13}$ accounts for the isospin asymmetry of ${ }^{139} \mathrm{La}$. The energy dependence of the ratio $\left\langle n_{\pi}\right\rangle / A$ is displayed in Fig. 2. In addition to the data at 530, 740, and $1350 \mathrm{MeV} /$ nucleon shown in Fig. 1 , the results of central-trigger runs at 990 and 1200 $\mathrm{MeV} /$ nucleon are plotted from an earlier run when the downstream scintillator array was not available. The mean numbers of participant nucleons at these two energies were interpolated from the $740-$ and 1350$\mathrm{MeV} /$ nucleon data where identical central-trigger cross sections were maintained. Also shown in Fig. 2 are previous results ${ }^{6}$ for the $\mathrm{Ar}+\mathrm{KCl}$ system. No significant difference between the $\mathrm{Ar}+\mathrm{KCl}$ and $\mathrm{La}+\mathrm{La}$ data is observed. The constancy of $\left\langle n_{\pi}\right\rangle / A$, both as the mass of the

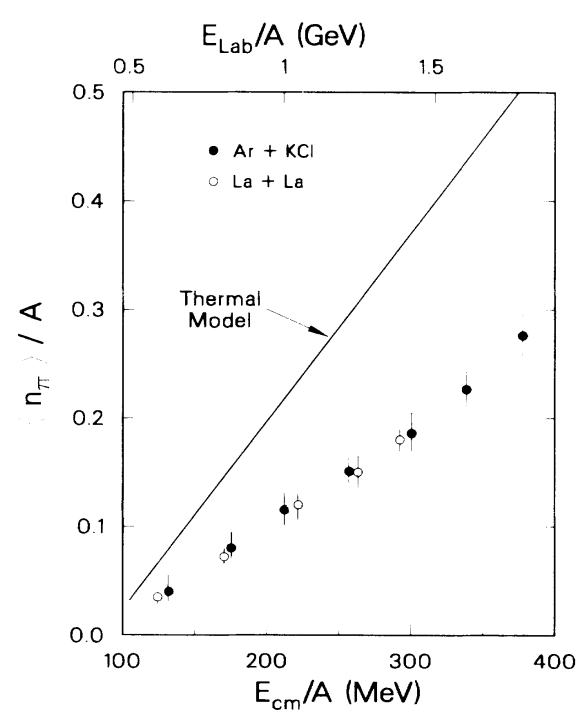

FIG. 2. The ratio of the mean pion multiplicity to the number of participant nucleons as a function of incident c.m. energy (bottom scale) and laboratory energy (top scale). Plotted are the La+La data points (open circles) and data from Ref. 6 for $\mathrm{Ar}+\mathrm{KCl}$ (dots). Also displayed is a thermal-model prediction (Ref. 14) which does not incorporate potential degrees of freedom. 
interacting nuclei is changed from $\mathrm{Ar}+\mathrm{KCl}$ to $\mathrm{La}+\mathrm{La}$ and as the interaction volume is changed by variation of the trigger conditions, demonstrates that pion production is a bulk nuclear-matter probe rather than a surface probe. An $A^{2 / 3}$ dependence ${ }^{15}$ of $\left\langle n_{\pi}\right\rangle$ is strictly ruled out by these data.

In the past we have suggested ${ }^{6}$ that the pion abundance per participant $\left\langle n_{\pi}\right\rangle / A$ is determined at the end of the high-density stage of the reaction before expansion, rendering $\left\langle n_{\pi}\right\rangle / A$ a sensitive probe of the compressed fireball, unaffected by the expansion phase. The mass independence of $\left\langle n_{\pi}\right\rangle / A$ supports that assumption since the expansion dynamics should depend strongly on mass. For example, the expansion rate, or volume doubling time, should increase as $A^{1 / 3}$. If the pion yield readjusts during expansion, a reduced $\left\langle n_{\pi}\right\rangle / A$ ratio would be expected in heavier systems, ${ }^{16}$ contrary to what is observed.

Assuming that the pion abundance reflects the thermal energy per baryon of the high-density stage, our results can be used to determine the thermal energy in a way that is independent of the dynamics of the collision process. Figure 2 includes the results of thermal-model calculations, ${ }^{14}$ for an equilibrium mixture of nucleons, deltas, and pions. The $\left\langle n_{\pi}\right\rangle / A$ ratio increases with the c.m. energy per nucleon, but with a steeper slope than the data. The reduced pion yield results from excitation of nonthermal degrees of freedom of the nuclear medium such as the potential energy stored in compression, and from off-shell mass effects. ${ }^{17}$ Medium influences on the observed pion yield are presently under investigation. ${ }^{18}$ Pending results of those studies, let us examine the consequences of the simple assumption that the main influence arises from conversion of kinetic into potential energy, implicit in our previous efforts ${ }^{5,6}$ to link the $\left\langle n_{\pi}\right\rangle / A$ ratio to the nuclear-matter equation of state.

The "missing" potential energy, inactive as far as particle production is concerned, can be determined from Fig. 2. It is the difference between the c.m. energy of the experiment, which is the total available energy, and the thermal energy which is the energy necessary in the thermal model to create the observed $\left\langle n_{\pi}\right\rangle / A$ ratio. The results are displayed in Fig. 3(a). Plotted is the missing energy per nucleon, $E^{\text {pot}} / A$, at each incident c.m. energy of the $\mathrm{Ar}+\mathrm{KCl}$ and $\mathrm{La}+\mathrm{La}$ experiments. $E^{\mathrm{pot}} / A$ is the increase of potential energy per participant nucleon expended in going from initial ground-state nuclear density $\rho_{0}$ to the density $\rho$ reached at each of the incident energies. In dynamical models this density $\rho\left(E_{\mathrm{c} . \mathrm{m} .} / A\right)$ is a monotonically increasing function of $E_{\mathrm{c} . \mathrm{m}} / A$. Note that the fraction of initial c.m. energy that is transformed into potential energy rises from $13 \%$ at $125 \mathrm{MeV} / \mathrm{nu}$ cleon to $33 \%$ at $380 \mathrm{MeV} /$ nucleon.

It is of specific theoretical interest to relate the potential energy to the nuclear density, within the limitations of the present approach. The cascade ${ }^{5}$ and the onedimensional Rankine-Hugoniot shock compression ${ }^{6}$

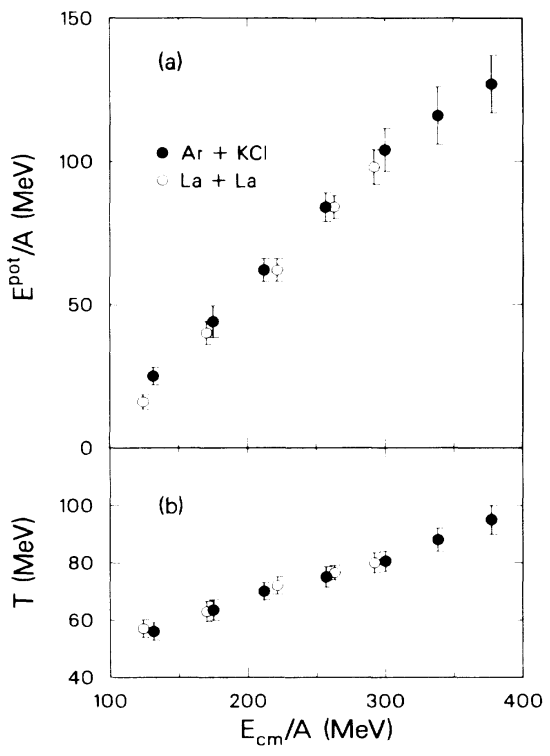

FIG. 3. (a) The potential energy as a function of c.m. energy derived from Fig. 2 as described in the text. (b) The fireball temperatures derived from the pion-multiplicity data and the thermal model (Ref. 14).

models were previously used to ascertain density $\left(\rho / \rho_{0}\right)$ at each bombaring energy. $E^{\text {pot }} A$ can be determined from the data, as shown above. The nuclear-matter energy-density relation is

$$
\begin{array}{r}
W\left(\rho / \rho_{0}\right)=\left(E^{\mathrm{pot} / A}\right)\left(\rho / \rho_{0}\right)+21\left[\left(\rho / \rho_{0}\right)^{2 / 3}-1\right] \\
+W\left(\rho / \rho_{0}=1\right),
\end{array}
$$

where the second term approximates ${ }^{19}$ the Fermi-energy contribution to the ground-state energy and the third, constant term is the binding energy at $\rho / \rho_{0}=1$. As dynamical models contribute additional uncertainty to the determination of $W\left(\rho / \rho_{0}\right)$ we do not pursue this result in detail. It is sufficient to note that all dynamical models $7,11,16,19$ predict nuclear density to reach a value of $\rho / \rho_{0}=3.5 \pm 0.5$ at $400 \mathrm{MeV} /$ nucleon c.m. energy. From Fig. 3(a) and Eq. (1) we then obtain the estimate $W=145 \pm 35 \mathrm{MeV}$ at $\rho / \rho_{0}=3.5$. This corresponds to an extremely "stiff" nuclear-matter equation of state compared to that used in nuclear-matter calculations at $T=0$, where $W\left(\rho / \rho_{0}=3.5\right)=40 \mathrm{MeV} .^{20}$

Baron and co-workers ${ }^{1}$ have recently argued that a significant fraction of the apparent stiffness of the equation of state in nuclear collisions should result from the high relative baryon velocities, characteristic of high densities and temperatures in the fireball. The temperatures reached in the fireball can be determined from the thermal energy per nucleon extracted from the data in Fig. 2. The corresponding temperatures are displayed in 
Fig. 3(b). They increase monotonically from 55 to 80 $\mathrm{MeV}$ over the energy range of the present experiment and up to $95 \mathrm{MeV}$ for $\mathrm{Ar}+\mathrm{KCl}$. A similar result has recently been reported by Hahn and Stöcker. ${ }^{21}$ These temperatures are to be distinguished clearly from "temperatures" extracted from the energy spectra which are affected by expansion dynamics, including flow, and for the pion by decay kinematics of the delta resonance.

In summary, we have investigated pion production for $\mathrm{La}+\mathrm{La}$ and found a linear dependence both on the participant nucleon number, which reflects fireball size, and on the incident energy. The relative pion abundance, $\left\langle n_{\pi}\right\rangle / A$, is the same for $140+140$ and $40+40$ collisions over the Bevalac energy range. This $A$ scaling of the pion multiplicity contradicts intuitive arguments concerning size-dependent absorption losses. Estimates of the fireball temperatures and the potential part of the compressional energy were presented. A very stiff nuclear-matter equation of state is predicted from nucleus-nucleus collisions.

The authors wish to acknowledge stimulating discussions with G. E. Brown, R. Malfliet, J. Molitoris, P. Siemens, and H. Stöcker. We also thank J. Brannigan and Bevalac Operations for their support during the experiments. This work was supported in part by the Director, Office of Energy Research, Division of Nuclear Physics of the Office of High Energy and Nuclear Physics of the U.S. Department of Energy under Contracts No. DEAC03-76SF00098 and No. DE-AS05-85ER40207.

(a) Present address: EP Division, CERN CH-1211, Geneva 23, Switzerland.

(b) Present address: Gesellschaft für Schwerionenforschung, Darmstadt, West Germany.
${ }^{1} \mathrm{E}$. Baron et al., to be published.

${ }^{2}$ E. Baron, J. Cooperstein, and S. Kahana, Nucl. Phys. A440, 744 (1985)

${ }^{3}$ J. M. Irvine, Progress in Particle and Nuclear Physics (Pergamon, Oxford, 1979), Vol. 2, p. 201.

${ }^{4}$ M. Nauenberg and G. Chapline, J. Astrophys. 179, 277 (1973).

${ }^{5}$ R. Stock et al., Phys. Rev. Lett. 49, 1236 (1982).

${ }^{6} \mathrm{~J}$. W. Harris et al., Phys. Lett. 153B, 377 (1985).

${ }^{7}$ H. Kruse, B. V. Jacak, and H. Stöcker, Phys. Rev. Lett. 54, 289 (1985); J. J. Molitoris, D. Hahn, and H. Stöcker, Nucl. Phys. A447, 13c (1985); J. Aichelin and C. M. Ko, Phys. Rev. Lett. 55, 2667 (1985).

${ }^{8}$ H. A. Gustafsson et al., Phys. Rev. Lett. 52, 1590 (1984).

${ }^{9}$ R. E. Renfordt et al., Phys. Rev. Lett. 53, 763 (1984); P. Danielewicz and G. Odyniec, Phys. Lett. 157B, 146 (1985).

${ }^{10}$ A. Sandoval et al., Phys. Rev. Lett. 45, 874 (1980).

${ }^{11} \mathrm{~J}$. Cugnon, T. Mitzutani, and J. Vandermeulen, Nucl. Phys. A352, 505 (1981); J. Cugnon, D. Kinet, and J. Vandermeulen, Nucl. Phys. A379, 553 (1982).

${ }^{12}$ For details of data-reduction techniques and efficiency calculations see W. Rauch, Ph.D. thesis, University of Frankfurt, 1986 (unpublished).

${ }^{13} \mathrm{~J}$. J. Molitoris, private communication of $\pi^{+} / \pi^{-} / \pi^{0}$ ratios from microscopic $V U U$ theory.

${ }^{14}$ J. Gosset, J. I. Kapusta, and G. D. Westfall, Phys. Rev. C 18, 844 (1978); G. D. Westfall, private communication.

${ }^{15}$ S. Nagamiya and M. Gyulassy, Advances in Nuclear Physics (Plenum, New York, 1984), Vol. 13, p. 201.

${ }^{16}$ Only yields of equal-mass $m_{A}=m_{B}$ collisions should be compared at the same incident energies. The c.m. energies and temperatures are typically lower for $m_{A}<m_{B}$ collisions.

17 J. D. Walecka, Ann. Phys. (N.Y.) 83, 491 (1974).

${ }^{18} \mathrm{~W}$. Botermans and R. Malfliet, Phys. Lett. 171B, 22 (1986); P. Siemens, private communication.

${ }^{19}$ M. Sano et al., Phys. Lett. 156B, 27 (1985).

${ }^{20}$ B. Friedman and V. R. Pandharipande, Nucl. Phys. A361, 502 (1981).

${ }^{21}$ D. Hahn and H. Stöcker, Nucl. Phys. A452, 723 (1986). 